OPEN ACCESS

Edited by:

Tianyu Zhang,

Guangzhou Institutes of Biomedicine and Health, Chinese Academy of Sciences (CAS), China

Reviewed by:

Meng Rui Lee,

National Taiwan University, Taiwan

Qi Guo,

Tongji University, China

*Correspondence:

Aimei Liu

liuaimeid@163.com

Yu Pang

pangyupound@163.com

${ }^{\dagger}$ These authors have contributed equally to this work

Specialty section:

This article was submitted to Infectious Agents and Disease,

a section of the journal

Frontiers in Microbiology

Received: 09 November 2021 Accepted: 15 December 2021

Published: 31 January 2022

Citation:

Huang L, Li H, Ren W, Zhang $X$, Shang Y, Liu Y, Liu A and Pang Y

(2022) Highly Discriminative

Genotyping of Mycobacterium abscessus Complex Using a Set of Variable Number Tandem Repeats in China. Front. Microbiol. 12:802133.

doi: 10.3389/fmicb.2021.802133

\title{
Highly Discriminative Genotyping of Mycobacterium abscessus Complex Using a Set of Variable Number Tandem Repeats in China
}

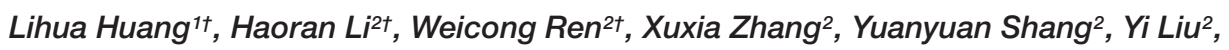
Aimei Liu ${ }^{1 *}$ and Yu Pang ${ }^{2 *}$

${ }^{1}$ Longtan Hospital of Guangxi Zhuang Autonomous Region, Liuzhou, China, ${ }^{2}$ Department of Bacteriology and Immunology, Beijing Chest Hospital, Beijing Tuberculosis and Thoracic Tumor Research Institute, Capital Medical University, Beijing, China

In this study, our aims were to comparatively analyze the power of variable number tandem repeat (VNTR) typing to discriminate isolates within subspecies and to identify a potential genetic marker for better molecular typing of Mycobacterium abscessus complex (MABC) strains. A total of 103 clinical MABC isolates were collected from a nationwide cross-sectional study in China. Eighteen VNTR loci were chosen to genotype the MABC isolates. Of the 103 clinical MABC isolates, there were 76 (73.8\%) M. abscessus subsp. abscessus (MAA) and 27 (26.2\%) M. abscessus subsp. massiliense (MAM) isolates. Among the patients with MAA lung diseases, the percentage of patients older than 45 years $(67.1 \%)$ was significantly higher than that of patients with MAM lung diseases [33.3\%, adjusted odds ratio $(\mathrm{aOR})=0.36,95 \%$ $\mathrm{Cl}=0.13-0.98, p=0.046]$. Fifteen VNTR loci were designated as being "highly discriminant" in our sample, except for TR109. The total of 103 MABC isolates were fully discriminated into 103 unique patterns by an 18-locus VNTR set [Hunter-Gaston Discriminatory Index $(\mathrm{HGDI})=1.000]$, of which the inclusion of the top 12 loci yielded a comparative $\mathrm{HGDI}$ value ( $\mathrm{HGDI}=0.9998)$. Remarkably, the order of the diversity of the VNTR loci showed significant difference between the MAA and MAM isolates. TR137 and TR2, two loci with high diversity indices for the MAA isolates, only yielded poor discriminatory power for the MAM isolates; the allelic diversity $(h)$ values were 0.0000 and 0.2621, respectively. A detailed analysis of TR137 in combination with the other 17 VNTR loci showed that the combination of TR137-TR2 could fully distinguish MAA from MAM isolates. In conclusion, our data revealed that MAA is more prone to affect elderly patients. Additionally, the population structure of the MABC isolates circulating in China has high diversity. The combined use of the TR137 and TR2 loci provides a simple criterion for the precise identification of MABC to the subspecies level.

Keywords: Mycobacterium abscessus complex, subspecies, polymorphism, genotyping, MIRU-VNTR 


\section{INTRODUCTION}

Worldwide, infections due to non-tuberculous mycobacteria (NTM) appear to be increasing (Thomson and NTM working group at Queensland TB Control Centre and Queensland Mycobacterial Reference Laboratory, 2010; Pang et al., 2017). Despite controversy as to the human-to-human transmission of NTM, their ubiquitous distribution in the environment increases the risk of NTM diseases in humans, especially in immunosuppressed and immunocompetent individuals (Bange et al., 2001; Ricketts et al., 2014; Harris et al., 2015; McShane and Glassroth, 2015). Approximately 200 NTM species have been identified, of which the Mycobacterium avium complex (MAC), Mycobacterium kansasii, and the Mycobacterium abscessus complex (MABC) are the major causative agents of human NTM diseases (Johansen et al., 2020). Significant differences in the geographic of NTM have been noted, reflecting diversities of their distribution in environmental niche across geographical locations (Pang et al., 2017).

MABC is composed of the most common rapid growing mycobacteria that cause human diseases, accounting for $23.1 \%$ of pulmonary infections due to NTM in China (Tan et al., 2021). It can also affect multiple organs and lead to severe respiratory, skin, and mucosal infections in humans (Ryan and Byrd, 2018; Kwak et al., 2019). More importantly, clinical treatment of MABC infections remains difficult, as it is inherently resistant to the majority of available antibiotics (Nessar et al., 2012; Nie et al., 2014; Lopeman et al., 2019). Patients infected with $\mathrm{MABC}$ are generally associated with poor clinical outcomes, particularly individuals with impaired immunity and underlying lung diseases. MABC comprises three closely related species: M. abscessus subsp. abscessus (MAA), M. abscessus subsp. massiliense (MAM), and M. abscessus subsp. bolletii (MAB) (Johansen et al., 2020). Despite sharing a high sequence similarity within MABC subspecies, a clinically significant difference has been observed between MAA and MAM, which was primarily caused by the clarithromycin (CLA) resistance-associated gene erm(41) (Kim et al., 2010; Brown-Elliott et al., 2015). MAM has a truncated erm(41) gene, whereas MAA has a functional erm(41) gene corresponding to inducible resistance, thereby leading to poorer outcomes (Choi et al., 2012). This sequence divergence indicates significant evolutionary differences between these two neighboring mycobacteria. Further comparative investigation of their genetic diversity will extend our knowledge of these microorganisms.

Variable number tandem repeat (VNTR) analysis has been widely accepted as an alternative to the differentiation of mycobacteria strains (Wong et al., 2012; Iakhiaeva et al., 2013; Zhao et al., 2014). For MABC, an 18-locus VNTR combination has been well established for genotyping strains belonging to this species and has shown promisingly good discriminatory power with a panel of MABC strains isolated from NTM pulmonary disease (NTM-PD) patients (Wong et al., 2012). In the study described here, we extended that initial study by applying VNTR typing to a large strain panel comprising MAA and MAM strains collected from a national surveillance in China. Our aims "were to comparatively analyze the power of VNTR typing to discriminate isolates within these subspecies and to identify a potential genetic marker for better molecular typing of MABC strains.

\section{MATERIALS AND METHODS}

\section{Bacterial Strains}

A total of 103 clinical MABC isolates were collected from the China Non-tuberculous Mycobacteria Surveillance Study (CNTMS), a nationwide cross-sectional study in China consecutively enrolling smear-positive patients with clinical symptoms suggestive of tuberculosis from December 2019 to June 2020 (Tan et al., 2021). The demographic and clinical variables were obtained from the electronic patient records in each hospital.

All NTM isolates were transferred to the Beijing Chest Hospital, Capital Medical University, for further analysis. Prior to species identification, the isolates were recovered on LöwensteinJensen (L-J) medium for 7 days.

\section{Species Identification}

Fresh colonies were harvested from the surface of the L-J medium with a plastic loop. A bacterial suspension of McFarland 1.0 standard was prepared for genomic DNA extraction using the cetyltrimethylammonium bromide (CTAB) $\mathrm{NaCl}$ method as previously described (Devonshire et al., 2015). Multilocus sequence analysis was used as the gold standard to differentiate the MABC isolates into the subspecies level. Primary identification to the species level was performed by ruling out membership of the NTM species using the MeltPro ${ }^{\circledR}$ Mycobacteria Identification Kit (Zeesan Biotech, Xiamen, China) (Xu et al., 2019), followed by sequencing of the entire rpoB and $h s p 65$ genes for the differentiation of the MABC isolates. The following primer pairs were used for PCR amplification as previously reported: $r p o B$ forward: 5'-CTA GCG GTA GTC GCT GTA GC-3', rpoB reverse: $5^{\prime}$-GTG CTC GAC GTC AAC TTC TT- $3^{\prime}$; $h s p 65$ forward; $5^{\prime}$-ATG GCC AAG ACA ATT GCG TA-3', hsp65 reverse: 5'-TTA GAA GTC CAT GCC ACC CA3'. After purification with the QIAEXII Gel Extraction Kit (Qiagen, Hilden, Germany), the amplicons were sent to the Tsingke Company (Beijing, China) for sequencing service. DNA sequences were blasted with the homologous sequences of the reference mycobacterial strains using the Basic Local Alignment Search Tool (BLAST) from $\mathrm{NCBI}^{1}$. The DNA sequences of $r p o B$ and $h s p 65$ in this study have been submitted to GenBank.

\section{Genotyping}

Eighteen VNTR loci were chosen to genotype the MABC isolates in this study (Wong et al., 2012). The primers for VNTR loci were synthesized by the Tsingke Company (Beijing, China). The PCR reaction mixture was prepared as follows: $10 \mu \mathrm{l} 2 \times$ Taq PCR mixture (TIANGEN, Beijing, China), $0.5 \mu \mathrm{M}$ each primer, and $2.5 \mu \mathrm{l}$ genomic DNA. The final volume was adjusted to $20 \mu \mathrm{l}$ with distilled water, and the reaction mixture was then amplified

\footnotetext{
${ }^{1}$ http://www.ncbi.nlm.nih.gov/BLAST
} 
TABLE 1 | Differences in the characteristics between Mycobacterium abscessus subsp. abscessus (MAA) and M. abscessus subsp. massiliense (MAM).

\begin{tabular}{|c|c|c|c|c|c|c|c|}
\hline Characteristics & Total, $n=103(\%)$ & MAA, $n=76(\%)$ & MAM, $n=27(\%)$ & OR (95\% Cl) & $p$-value & Adjusted OR (95\% Cl) & $p$-value \\
\hline \multicolumn{8}{|l|}{ Sex } \\
\hline Male & 48 (46.6) & $40(52.6)$ & $8(29.6)$ & 1 & References & 1 & References \\
\hline Female & $55(53.4)$ & $36(47.4)$ & $19(70.4)$ & $2.64(1.03-6.76)$ & 0.04 & $1.86(0.68-5.13)$ & 0.229 \\
\hline \multicolumn{8}{|l|}{ Age (years) } \\
\hline $16-44$ & $43(41.7)$ & 25 (32.9) & $18(66.7)$ & 1 & References & 1 & References \\
\hline$\geq 45$ & $60(58.3)$ & $51(67.1)$ & 9 (33.3) & $0.25(0.10-0.62)$ & 0.002 & $0.36(0.13-0.98)$ & 0.046 \\
\hline \multicolumn{8}{|l|}{ Ethnicity } \\
\hline Han & $98(95.1)$ & $73(96.1)$ & $25(92.6)$ & 1 & References & - & - \\
\hline Other & $5(4.9)$ & 3 (3.9) & $2(7.4)$ & 1.95 (0.31-12.33) & 0.604 & - & - \\
\hline \multicolumn{8}{|c|}{ Previous TB episode } \\
\hline Yes & $87(84.5)$ & $64(84.2)$ & $23(85.2)$ & 1 & References & - & - \\
\hline No & $16(15.5)$ & $12(15.8)$ & $4(14.8)$ & $0.93(0.27-3.17)$ & 1 & - & - \\
\hline \multicolumn{8}{|l|}{ Comorbidity } \\
\hline Yes & 39 (37.9) & $34(44.7)$ & 5 (18.5) & 1 & References & 1 & References \\
\hline No & $64(62.1)$ & 42 (55.3) & $22(81.5)$ & $3.56(1.22-10.40)$ & 0.016 & $0.40(0.13-1.24)$ & 0.112 \\
\hline \multicolumn{8}{|l|}{ Symptoms } \\
\hline Cough & $80(77.7)$ & $57(75.0)$ & $23(85.2)$ & $1.92(0.59-6.25)$ & 0.42 & - & - \\
\hline Fever & $16(15.5)$ & $14(18.4)$ & $2(7.4)$ & 0.35 (0.08-1.67) & 0.227 & - & - \\
\hline Hemoptysis & $14(13.6)$ & $12(15.8)$ & $2(7.4)$ & $0.43(0.65-1.08)$ & 0.346 & - & - \\
\hline
\end{tabular}

TB, tuberculosis.

with initial denaturation at $94^{\circ} \mathrm{C}$ for $5 \mathrm{~min}$, followed by 35 amplification cycles of denaturation at $94^{\circ} \mathrm{C}$ for $30 \mathrm{~s}$, annealing at $62^{\circ} \mathrm{C}$ for $30 \mathrm{~s}$, and extension at $72^{\circ} \mathrm{C}$ for $30 \mathrm{~s}$, and finally extended at $72^{\circ} \mathrm{C}$ for $7 \mathrm{~min}$. The PCR products were electrophoresed in $2 \%$ agarose gel stained with 4 S Green Plus (TaKaRa, Shiga, Japan). The reference MAA strain (ATCC 19977) was run as an additional control of accuracy and for the accurate interpretation of the repeat number of each VNTR locus. The repeat numbers of different alleles were calculated according to the allele size range and the basic unit length.

\section{Statistical Analysis}

The discrimination ability of each tandem repeat (TR) was calculated and analyzed using Hunter-Gaston Discriminatory Index (HGDI) scores according to the method by Hunter and Gaston (1988). Data of the 18 TR loci of the MABC isolates were analyzed using the BioNumerics software (version 5.0; Applied Maths, Sint-Martens-Latem, Belgium). A dendrogram was generated in BioNumerics using the average linkage clustering method (unweighted pair group method with arithmetic mean, UPGMA). One-way ANOVA was used to compare the differences between multiple groups. The difference was considered statistically significant when $p<0.05$. All calculations were performed using SPSS 22.0 software for Windows (SPSS Inc., Chicago, IL, United States).

\section{RESULTS}

\section{Demographic and Clinical Characteristics of Patients}

Among the 103 clinical MABC isolates circulating in China, $76(73.8 \%)$ were MAA and $27(26.2 \%)$ were MAM isolates.
The baseline demographic and clinical characteristics are shown in Table 1. Of the patients, 53.4\% (55/103) were females, and patients aged $>45$ years $(60 / 103)$ accounted for the majority of the studied participants. In addition, 87 cases $(84.5 \%)$ had previous tuberculosis (TB) episodes. We further compared the percentages of the demographic and clinical characteristics of patients with MAA and MAM lung diseases. Among the patients with MAA lung diseases, the percentage of patients older than 45 years $(67.1 \%)$ was significantly higher than that of patients with MAM lung diseases [33.3\%, adjusted odds ratio $(\mathrm{aOR})=0.3695 \% \mathrm{CI}=0.13-0.98, p=0.046]$. In contrast, multiple logistic regression analysis revealed no statistically significant differences in several other demographic and clinical characteristics $(p>0.05)$.

\section{Allelic Diversity of the MABC Isolates and Distribution of Variable Number Tandem Repeat Allele Numbers}

Allelic diversity $(h)$ is a good index of the discriminatory power provided by each VNTR locus. On the basis of this index, 15 VNTR loci were designated as being "highly discriminant" $(h>0.6)$ in our sample, except for TR109, which was designated as moderately discriminant $(0.3 \leq h \leq 0.6$; Table 2$)$. The total of $103 \mathrm{MABC}$ isolates were fully discriminated into 103 unique patterns by the 18-locus VNTR set (HGDI = 1.000; Figure 1), of which the inclusion of the top 12 loci yielded a comparative HGDI value (HGDI = 0.9998).

\section{Subspecies Diversity of the Variable Number Tandem Repeat Loci}

We further analyzed the discriminatory power of each VNTR locus stratified to different subspecies (Figure $\mathbf{2}$ and 
TABLE 2 | Combined HGDI for 103 independent Mycobacterium abscessus complex (MABC) isolates.

\begin{tabular}{|c|c|c|c|c|c|c|c|c|c|c|c|c|c|c|c|c|c|c|c|c|}
\hline \multirow[t]{2}{*}{ Locus } & \multicolumn{13}{|c|}{ No. of isolates with VNTR repeat units } & \multirow[t]{2}{*}{ HGDI ${ }^{a}$} & \multicolumn{6}{|c|}{ Combined HGDIb } \\
\hline & 0 & 1 & 2 & 3 & 4 & 5 & 6 & 7 & 8 & 9 & 10 & 11 & 16 & & TR86 to TR172 & TR86 to TR116 & TR86 to TR200 & TR86 to TR131 & TR86 to TR101 & All loci \\
\hline TR86 & & 18 & 27 & 19 & 16 & 11 & 4 & 1 & 0 & 2 & 4 & 1 & & 0.8357 & 0.9882 & 0.9967 & 0.9998 & 1.0000 & 1.0000 & 1.0000 \\
\hline TR155 & 14 & 23 & 30 & 22 & 10 & 1 & 1 & 2 & & & & & & 0.7990 & & & & & & \\
\hline TR172 & & 6 & 15 & 27 & 26 & 24 & 2 & 2 & 1 & & & & & 0.7955 & & & & & & \\
\hline TR167 & & & 25 & 30 & 29 & 7 & 8 & 2 & 1 & 1 & & & & 0.7733 & & & & & & \\
\hline TR116 & 1 & 28 & 26 & 26 & 19 & 0 & 3 & & & & & & & 0.7712 & & & & & & \\
\hline TR45 & 1 & 10 & 36 & 30 & 12 & 2 & 3 & 2 & 7 & & & & & 0.7712 & & & & & & \\
\hline TR163 & 13 & 22 & 34 & 26 & 7 & 0 & 1 & & & & & & & 0.7685 & & & & & & \\
\hline TR150 & 9 & 27 & 37 & 15 & 11 & 4 & & & & & & & & 0.7679 & & & & & & \\
\hline TR137 & & 7 & 40 & 19 & 15 & 18 & 3 & 1 & & & & & & 0.7653 & & & & & & \\
\hline TR149 & 11 & 23 & 27 & 34 & 8 & & & & & & & & & 0.7624 & & & & & & \\
\hline TR2 & & 18 & 42 & 5 & 9 & 19 & 9 & 1 & & & & & & 0.7588 & & & & & & \\
\hline TR200 & & 11 & 29 & 40 & 9 & 6 & 4 & 3 & & & & & 1 & 0.7523 & & & & & & \\
\hline TR179 & 14 & 5 & 33 & 37 & 7 & 7 & & & & & & & & 0.7455 & & & & & & \\
\hline TR28 & & 12 & 37 & 44 & 10 & & & & & & & & & 0.6720 & & & & & & \\
\hline TR139 & & 19 & 57 & 21 & 4 & 1 & 0 & 1 & & & & & & 0.6225 & & & & & & \\
\hline TR131 & & 8 & 41 & 48 & 6 & & & & & & & & & 0.6210 & & & & & & \\
\hline TR101 & 4 & 15 & 58 & 26 & & & & & & & & & & 0.6023 & & & & & & \\
\hline TR109 & & & 4 & 42 & 50 & 7 & & & & & & & & 0.5978 & & & & & & \\
\hline
\end{tabular}

${ }^{a} \mathrm{HGDI}$, Hunter-Gaston Discriminatory Index for each variable number tandem repeat (NNTR) locus.

${ }^{b}$ The shaded portions show the combined HGDI values for TR86-TR172, TR86-TR116, TR86-TR200, TR86-TR131, and TR86-TR101, showing increasing discriminatory power from 0.9882 for 3 loci combined to a maximum of 1.0000 for 16 loci combined (matching the overall HGDI of 0.9563 for all 18 tandem repeats (TRs) combined, as shown). 


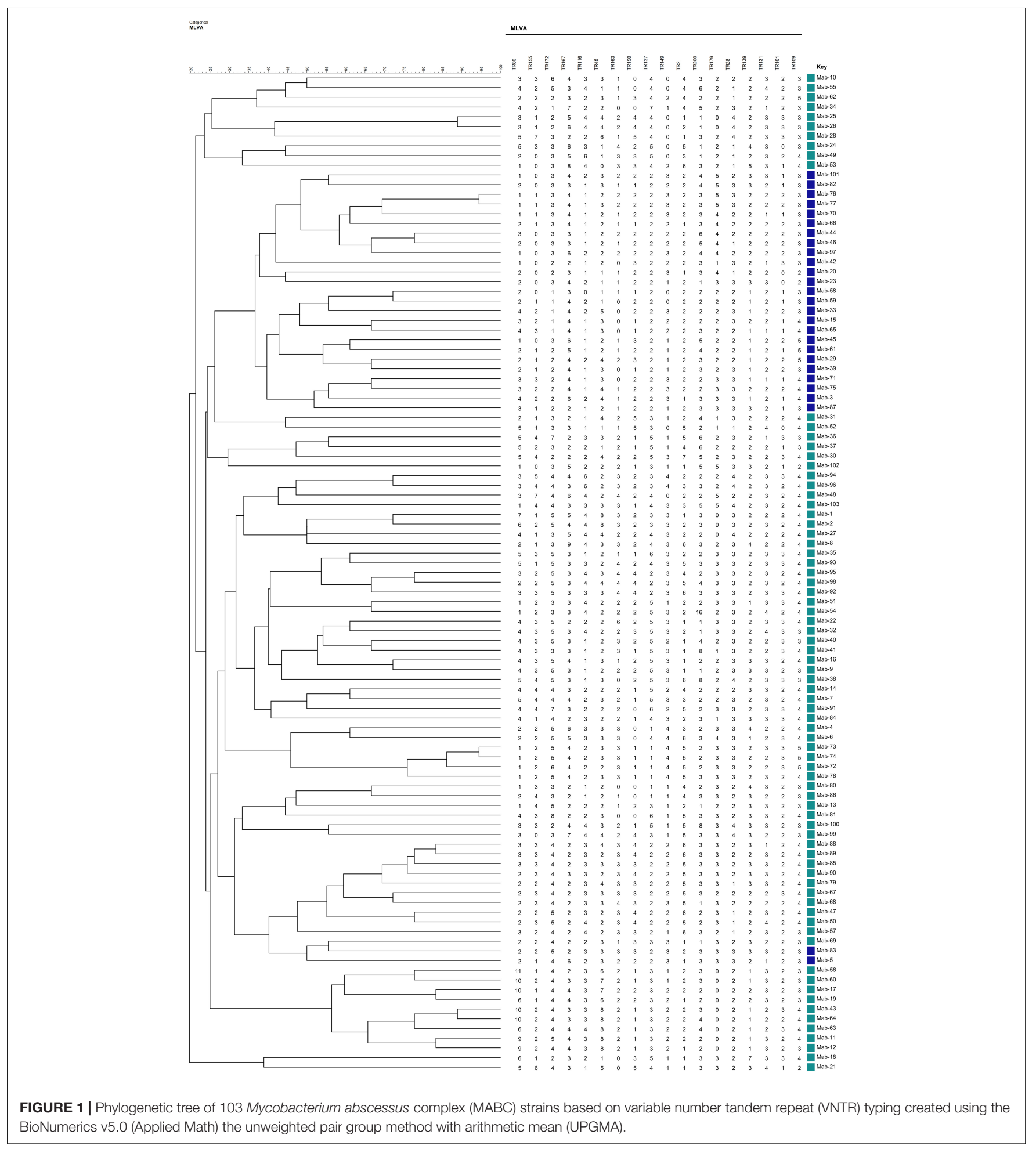

Supplementary Tables 1, 2). For the MAA isolates, the majority of the VNTR loci showed high-moderate diversity indices, consisting of TR86, TR2, and TR137. Four VNTR loci (TR101, TR109, TR131, and TR139) achieved a moderate diversity index. For the MAM isolates, high and moderate diversity indices were recorded in 11 and 5 VNTR loci, respectively. Remarkably, the order of the diversity of the VNTR loci showed significant difference between the MAA and MAM isolates. TR137 and TR2, two loci with high diversity indices for the MAA isolates, only yielded poor discriminatory power for the MAM isolates; the $h$ values were 0.0000 and 0.2621 , respectively. The repeat number of the MAM isolates at both TR2 and TR137 loci was less than 3. 


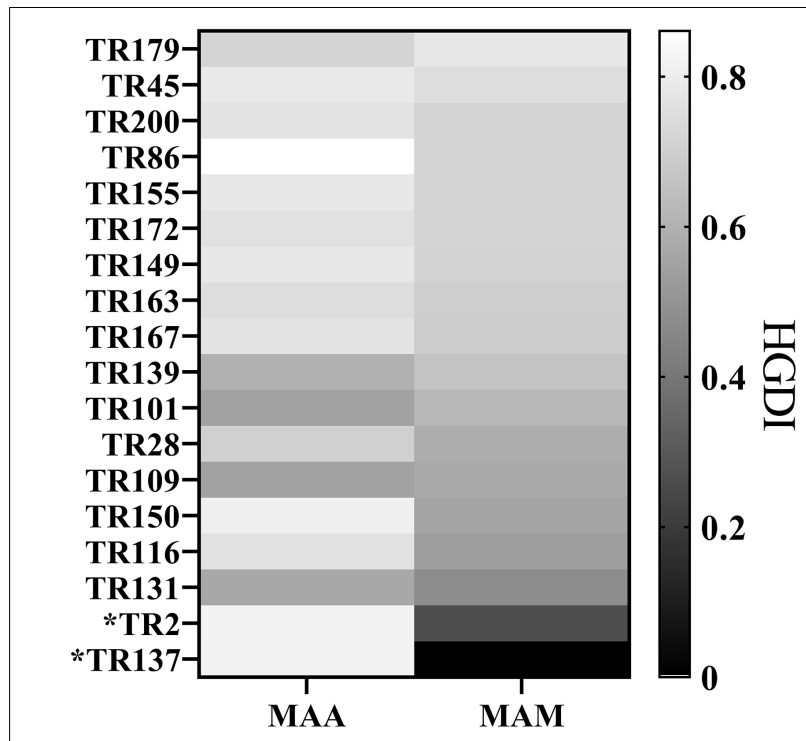

FIGURE 2 | Hunter-Gaston Discriminatory Index (HGDI) for each variable number tandem repeat (VNTR) locus stratified to different subspecies showing that the TR137 and TR2 loci exhibited the potential to distinguish subspecies within Mycobacterium abscessus complex (MABC).

Notably, all MAM isolates had two repeats at the TR137 locus, whereas the repeat number of the MAA isolates at this locus ranged from 1 to 7 , indicating that TR137 exhibited subspecies specificity within MABC.

\section{Accuracy of TR137 Plus TR2 for Species Identification Among MABC Isolates}

Subsequently, the potential VNTR locus combination that could be used for species identification was investigated. A detailed analysis of TR137 combined with the other 17 VNTR loci showed that the TR137-TR2 combination could fully distinguish MAA from MAM isolates (Figure 3). As shown in Figure 4, the majority of MAA with a TR137 repeat unit higher than 2 could be identified by TR137 screening. Thereafter, the remaining MABC isolates with a TR137 repeat unit equal to or lower than 2 were completely distinguished into MAA (TR2 repeat units $>2$ ) and MAM (TR2 repeat units $\leq 2$ ) by TR2 screening.

\section{DISCUSSION}

MABC has attracted increasing attention due to being the most chemotherapy-resistant member of NTM (Johansen et al., 2020). As the second prevalent NTM species in China, MABC accounts for $23.1 \%$ of patients with NTM-PD, of which $56.9 \%$ were MAA and the other $43.1 \%$ were MAM (Pang et al., 2017; Tan et al., 2021). We firstly identified the difference in age distribution between MAA and MAM. Compared with individuals infected with MAM, those with MAA were more frequently $>45$ years old. Considering that human immunity is known to significantly decline with increasing age (Valiathan et al., 2016), MAA seems to be less virulent than MAM. In line with our hypothesis, previous clinical data found that only onethird of MAA pulmonary infection presented obvious symptoms, whereas all patients with MAM pulmonary infection had obvious symptoms (Shin et al., 2013), indicating possible differences in the virulence between these neighboring subspecies. However, the reliability of the hypothesis is limited by the small cohort size included in the present study. An experimental investigation is needed to determine whether any differences in host responses and clinical outcomes exist among animals infected with these subspecies.

Our genotyping data with the classical 18-locus VNTR set revealed that the population structure of the MABC isolates circulating in China has high diversity. On one hand, we evaluated the optimal VNTR locus combination for genotyping MABC isolates. A comprehensive analysis of the genotyping data revealed that the inclusion of the top 12 loci yielded a comparative HGDI value (HGDI $=0.9998)$ relative to that of the 18-locus set (HGDI = 1.0000). Considering labor and cost, this 12-locus set may be a cost-effective tool for differentiating the MABC isolates in China. On the other hand, the high diversity of the MAC isolates may be attributed to their widespread geographic distribution across China, which were lacking of epidemiological links. Besides, our preliminary data support that there was no person-to-person transmission among the general population, despite potential nosocomial transmission of MABC occurring in cystic fibrosis patients in the United Kingdom (Bryant et al., 2013). The low risk of transmission of $\mathrm{MABC}$ in the community reflects the attenuated virulence of this NTM species. In addition, NTM species are well equipped to survive in the environment. Notably, MAC could survive in amoeba trophozoites and subsequent cyst stages (Drancourt, 2014). Its remarkable survival in amoeba would provide benefits for the transition of MABC from environmental organisms to human pathogens, thereby accelerating the accumulation of genetic diversity. Taken together, our data demonstrate that the MABC isolates causing pulmonary diseases primarily originate from the environmental niche. Further comparative investigation on environmental $\mathrm{MABC}$ isolates will extend our knowledge on the evolutionary pathway of MABC through the acquisition of important virulence genes.

Another interesting finding in our study was that the repeat numbers of TR137 exhibited significant species diversity between MAA and MAM. TR137 was present as one to seven copies in MAA, while MAM showed one pattern with two copies of TR137. Upon combing the genotyping results of TR2, another VNTR locus with discriminatory power for resolving subspecies, complete identity agreement was found between this novel assay and the reference method. Similar to our observation, Choi and colleagues found that two TR loci could be used to discriminate MAA and MAM via different copy numbers of VNTR11 and VNTR23 (Choi et al., 2011). In addition, our results suggest that genotypes in particular VNTR targets represent an evolution marker of the microdiversity of MABC, thus being involved in the pathogenesis of MAA and MAM lung diseases (Shin et al., 2013). The combined use of the TR137 and TR2 loci provides a simple criterion for the precise identification of MABC to 


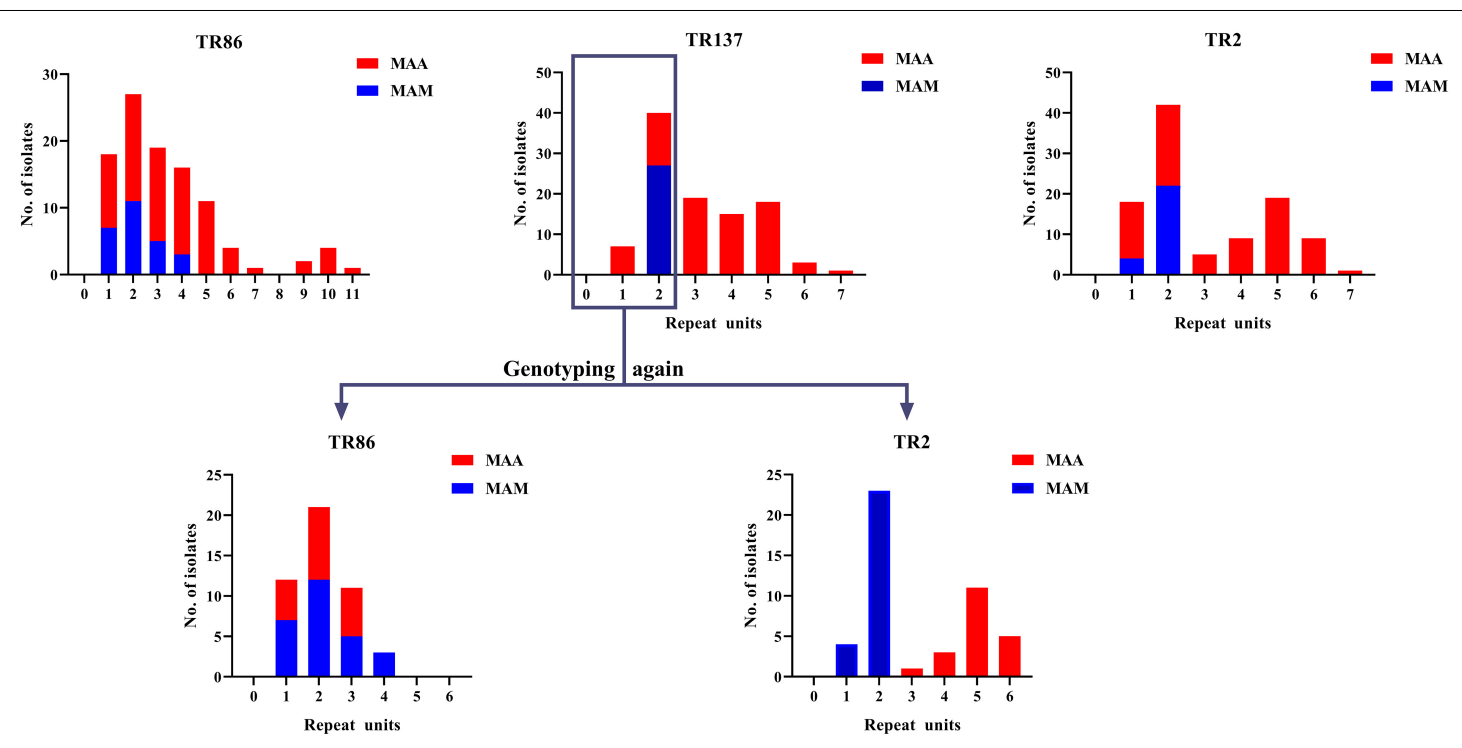

FIGURE 3 | Statistical analysis of Mycobacterium abscessus subsp. abscessus (MAA) and M. abscessus subsp. massiliense (MAM) repeats units at 18 tandem repeat (TR) loci showing that the repeat units of the MAM isolates were less than 3 only at TR137 and TR2 of the 18 TR loci (top). When the strains with TR137 repeat units less than 3 were detected again, only the TR2 locus could completely distinguish MAM from MAA (bottom).

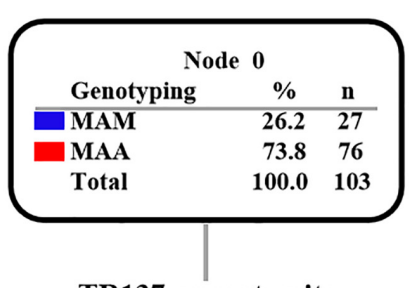

TR137 repeat units
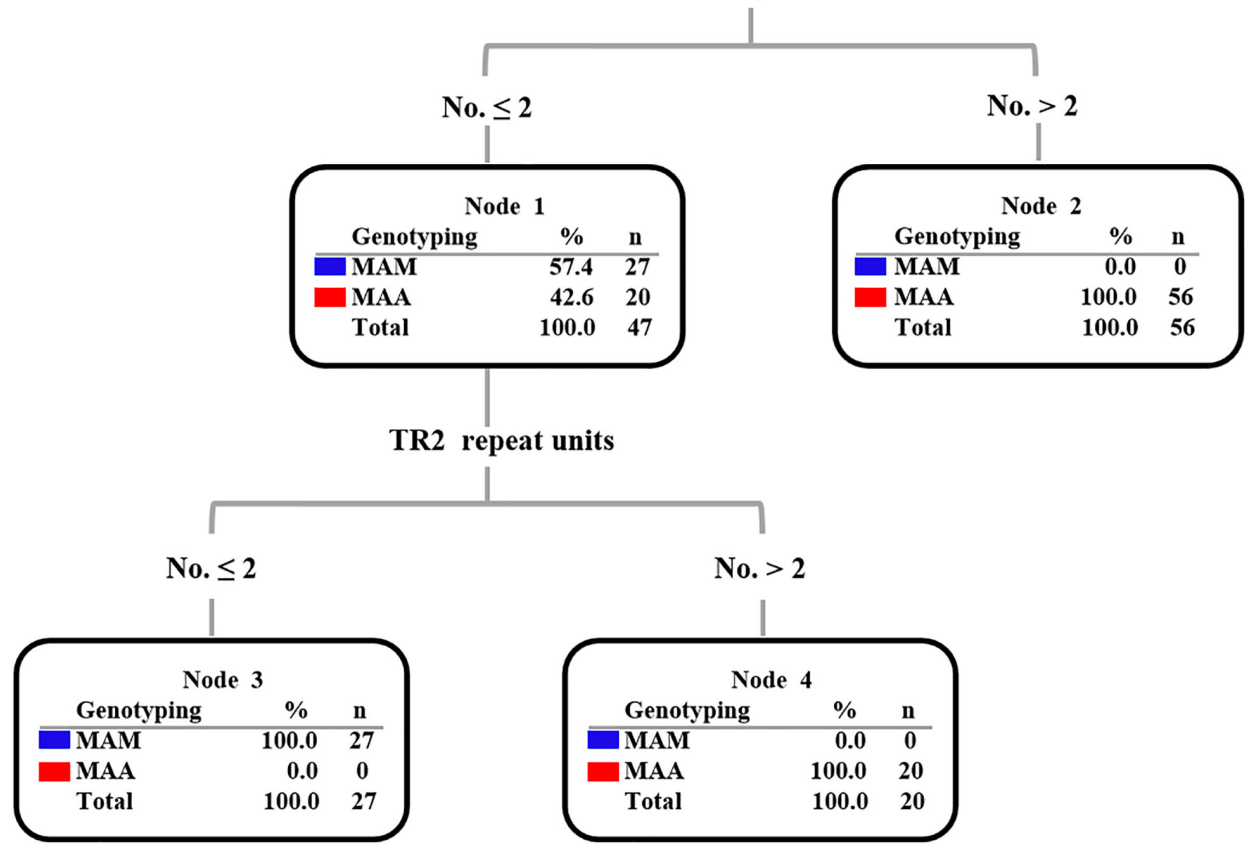

FIGURE 4 | Decision tree diagram based on tandem variable number tandem repeat (VNTR) showing that TR137 combined with the TR2 locus can distinguish Mycobacterium abscessus subsp. abscessus (MAA) and M. abscessus subsp. massiliense (MAM) in the Mycobacterium abscessus complex (MABC). 
the subspecies level. Compared with the conventional Sanger sequencing or other molecular methods, it only requires standard PCR and gel electrophoresis facilities rather than expensive equipment. Besides, with a 4 -h procedure time and a reagent cost of United States \$3, this method seems affordable and could be easily available in routine microbiology laboratories, especially laboratories in resource-limited settings.

We also acknowledge several obvious limitations of the present study. Firstly, despite the enrollment of all MABC isolates collected in the national surveillance, the small sample size may limit the confidence of our conclusion. Further validation of this novel two-locus VTNR set in a larger series of MABC patients is required to validate our findings. Secondly, another important subspecies, M. abscessus subsp. bolletii (MAB), was not identified in the NTM isolates from China, which was inconsistent with our previous observations (Pang et al., 2017). As a consequence, our observation should be confirmed in clinical MAB isolates. Thirdly, clarithromycin susceptibility was not determined in this study, which hampered further analysis of the association between the VNTR pattern and the minimal inhibitory concentration (MIC) of clarithromycin. Lastly, a previous report revealed that the MABC genotype influenced mycobacterial virulence (Shin et al., 2013), thus presenting a remarkable difference in clinical disease phenotype and disease progression. Unfortunately, the clinical outcomes of the patients infected with MABC were not collected in this observational study. Further study is urgently required for elucidating the correlation between phenotype and genotype among the MABC isolates.

\section{CONCLUSION}

Our data revealed the difference in age distribution between MAA and MAM, and MAA was more prone to affect elderly patients. In addition, the population structure of the MABC isolates circulating in China showed a high diversity, indicating that the MABC isolates causing pulmonary diseases primarily originate from the environmental niche. The combined use of the TR137 and TR2 loci provided a simple criterion for the precise identification of MABC to the subspecies level. To extend these results, studies of the association between the clinical outcomes, distinct pathogenesis, and VNTR profiles are warranted.

\section{REFERENCES}

Bange, F. C., Brown, B. A., Smaczny, C., Wallace, R. J. Jr., and Bottger, E. C. (2001). Lack of transmission of mycobacterium abscessus among patients with cystic fibrosis attending a single clinic. Clin. Infect. Dis. 32, 1648-1650. doi: $10.1086 / 320525$

Brown-Elliott, B. A., Vasireddy, S., Vasireddy, R., Iakhiaeva, E., Howard, S. T., Nash, K., et al. (2015). Utility of sequencing the erm(41) gene in isolates of Mycobacterium abscessus subsp. abscessus with low and intermediate clarithromycin MICs. J. Clin. Microbiol. 53, 1211-1215. doi: 10.1128/JCM. 02950- 14

Bryant, J. M., Grogono, D. M., Greaves, D., Foweraker, J., Roddick, I., Inns, T., et al. (2013). Whole-genome sequencing to identify transmission of Mycobacterium abscessus between patients with cystic fibrosis: a retrospective

\section{DATA AVAILABILITY STATEMENT}

The datasets presented in this study can be found in online repositories. The names of the repository/repositories and accession number(s) can be found below: GenBank, OL704521 - OL704623, OL704624 - OL704726.

\section{ETHICS STATEMENT}

This study was approved by the Ethics Committee of Beijing Chest Hospital, Capital Medical University. Informed consent from all participating subjects was obtained.

\section{AUTHOR CONTRIBUTIONS}

YP, WR, and AL conceptualized the study. YP and YL designed the methodology. LH, YS, and XZ performed formal analysis. LH, $\mathrm{AL}$, and $\mathrm{XZ}$ conducted the investigation. HL, WR, and YS curated the data. HL, YP, and LH prepared the original draft. YP, AL, and $\mathrm{HL}$ reviewed and edited the manuscript. YL, YP, and AL acquired funding. All authors contributed to the manuscript and approved the submitted version.

\section{FUNDING}

The current work received support from the Beijing Hospitals Authority Ascent Plan (DFL20191601), Beijing Hospitals Authority Clinical Medicine Development of Special Funding (ZYLX202122), and Capital's Funds for Health Improvement and Research (2020-1-1041), which are Development, Popularization and Application of appropriate Medical and Health Technology in Guangxi Province (S2019035 and S2020065), and Liuzhou Science and Technology Plan Project (2020NBAD0802).

\section{SUPPLEMENTARY MATERIAL}

The Supplementary Material for this article can be found online at: https://www.frontiersin.org/articles/10.3389/fmicb. 2021.802133/full\#supplementary-material

cohort study. Lancet 381, 1551-1560. doi: 10.1016/S0140-6736(13) 60632-7

Choi, G. E., Chang, C. L., Whang, J., Kim, H. J., Kwon, O. J., Koh, W. J., et al. (2011). Efficient differentiation of Mycobacterium abscessus complex isolates to the species level by a novel PCR-based variable-number tandem-repeat assay. J. Clin. Microbiol. 49, 1107-1109. doi: 10.1128/JCM.02318-10

Choi, G. E., Shin, S. J., Won, C. J., Min, K. N., Oh, T., Hahn, M. Y., et al. (2012). Macrolide treatment for Mycobacterium abscessus and Mycobacterium massiliense infection and inducible resistance. Am. J. Respir. Crit. Care Med. 186, 917-925. doi: 10.1164/rccm.201111-2005OC

Devonshire, A. S., Honeyborne, I., Gutteridge, A., Whale, A. S., Nixon, G., Wilson, P., et al. (2015). Highly reproducible absolute quantification of Mycobacterium tuberculosis complex by digital PCR. Anal. Chem. 87, 3706-3713. doi: 10.1021/ ac5041617 
Drancourt, M. (2014). Looking in amoebae as a source of mycobacteria. Microb. Pathog. 77, 119-124. doi: 10.1016/j.micpath.2014.07.001

Harris, K. A., Underwood, A., Kenna, D. T., Brooks, A., Kavaliunaite, E., Kapatai, G., et al. (2015). Whole-genome sequencing and epidemiological analysis do not provide evidence for cross-transmission of mycobacterium abscessus in a cohort of pediatric cystic fibrosis patients. Clin. Infect. Dis. 60, 1007-1016. doi: $10.1093 / \mathrm{cid} / \mathrm{ciu} 967$

Hunter, P. R., and Gaston, M. A. (1988). Numerical index of the discriminatory ability of typing systems: an application of Simpson's index of diversity. J. Clin. Microbiol. 26, 2465-2466. doi: 10.1128/jcm.26.11.2465-2466.1988

Iakhiaeva, E., McNulty, S., Brown Elliott, B. A., Falkinham, J. O. III, Williams, M. D., Vasireddy, R., et al. (2013). Mycobacterial interspersed repetitive-unitvariable-number tandem-repeat (MIRU-VNTR) genotyping of mycobacterium intracellulare for strain comparison with establishment of a PCR-based database. J. Clin. Microbiol. 51, 409-416. doi: 10.1128/JCM.02443-12

Johansen, M. D., Herrmann, J. L., and Kremer, L. (2020). Non-tuberculous mycobacteria and the rise of Mycobacterium abscessus. Nat. Rev. Microbiol. 18, 392-407. doi: 10.1038/s41579-020-0331-1

Kim, H. Y., Kim, B. J., Kook, Y., Yun, Y. J., Shin, J. H., Kim, B. J., et al. (2010). Mycobacterium massiliense is differentiated from Mycobacterium abscessus and Mycobacterium bolletii by erythromycin ribosome methyltransferase gene (erm) and clarithromycin susceptibility patterns. Microbiol. Immunol. 54, 347353. doi: 10.1111/j.1348-0421.2010.00221.x

Kwak, N., Dalcolmo, M. P., Daley, C. L., Eather, G., Gayoso, R., Hasegawa, N., et al. (2019). M ycobacterium abscessus pulmonary disease: individual patient data meta-analysis. Eur. Respir. J. 54:1801991. doi: 10.1183/13993003.01991-2018

Lopeman, R. C., Harrison, J., Desai, M., and Cox, J. A. G. (2019). Mycobacterium abscessus: environmental Bacterium Turned Clinical Nightmare. Microorganisms 7:90. doi: 10.3390/microorganisms7030090

McShane, P. J., and Glassroth, J. (2015). Pulmonary Disease Due to Nontuberculous Mycobacteria: current State and New Insights. Chest 148, 1517-1527. doi: 10.1378/chest.15-0458

Nessar, R., Cambau, E., Reyrat, J. M., Murray, A., and Gicquel, B. (2012). Mycobacterium abscessus: a new antibiotic nightmare. J. Antimicrob. Chemother. 67, 810-818. doi: 10.1093/jac/dkr578

Nie, W., Duan, H., Huang, H., Lu, Y., Bi, D., and Chu, N. (2014). Species identification of Mycobacterium abscessus subsp. abscessus and Mycobacterium abscessus subsp. bolletii using rpoB and hsp65, and susceptibility testing to eight antibiotics. Int. J. Infect. Dis. 25, 170-174. doi: 10.1016/j.ijid.2014.02.014

Pang, Y., Tan, Y., Chen, J., Li, Y., Zheng, H., Song, Y., et al. (2017). Diversity of nontuberculous mycobacteria in eastern and southern China: a cross-sectional study. Eur. Respir. J. 49:1601429. doi: 10.1183/13993003.01429-2016

Ricketts, W. M., O'Shaughnessy, T. C., and van Ingen, J. (2014). Human-to-human transmission of Mycobacterium kansasii or victims of a shared source? Eur. Respir. J. 44, 1085-1087. doi: 10.1183/09031936.00066614
Ryan, K., and Byrd, T. F. (2018). Mycobacterium abscessus: shapeshifter of the Mycobacterial World. Front. Microbiol. 9:2642. doi: 10.3389/fmicb.2018.02642

Shin, S. J., Choi, G. E., Cho, S. N., Woo, S. Y., Jeong, B. H., Jeon, K., et al. (2013). Mycobacterial genotypes are associated with clinical manifestation and progression of lung disease caused by Mycobacterium abscessus and Mycobacterium massiliense. Clin. Infect. Dis. 57, 32-39. doi: 10.1093/cid/cit172

Tan, Y., Deng, Y., Yan, X., Liu, F., Tan, Y., Wang, Q., et al. (2021). Nontuberculous mycobacterial pulmonary disease and associated risk factors in China: a prospective surveillance study. J. Infect. 83, 46-53. doi: 10.1016/j.jinf.2021.05. 019

Thomson, R. M., and NTM working group at Queensland TB Control Centre and Queensland Mycobacterial Reference Laboratory (2010). Changing epidemiology of pulmonary nontuberculous mycobacteria infections. Emerg. Infect. Dis. 16, 1576-1583. doi: 10.3201/eid1610.091201

Valiathan, R., Ashman, M., and Asthana, D. (2016). Effects of Ageing on the Immune System: infants to Elderly. Scand. J. Immunol. 83, 255-266. doi: 10. 1111/sji.12413

Wong, Y. L., Ong, C. S., and Ngeow, Y. F. (2012). Molecular typing of Mycobacterium abscessus based on tandem-repeat polymorphism. J. Clin. Microbiol. 50, 3084-3088. doi: 10.1128/JCM.00753-12

Xu, Y., Liang, B., Du, C., Tian, X., Cai, X., Hou, Y., et al. (2019). Rapid Identification of Clinically Relevant Mycobacterium Species by Multicolor Melting Curve Analysis. J. Clin. Microbiol. 57, e01096-18. doi: 10.1128/JCM. 01096-18

Zhao, X., Wang, Y., and Pang, Y. (2014). Antimicrobial susceptibility and molecular characterization of Mycobacterium intracellulare in China. Infect. Genet. Evol. 27, 332-338. doi: 10.1016/j.meegid.2014.07.032

Conflict of Interest: The authors declare that the research was conducted in the absence of any commercial or financial relationships that could be construed as a potential conflict of interest.

Publisher's Note: All claims expressed in this article are solely those of the authors and do not necessarily represent those of their affiliated organizations, or those of the publisher, the editors and the reviewers. Any product that may be evaluated in this article, or claim that may be made by its manufacturer, is not guaranteed or endorsed by the publisher.

Copyright (c) 2022 Huang, Li, Ren, Zhang, Shang, Liu, Liu and Pang. This is an open-access article distributed under the terms of the Creative Commons Attribution License (CC BY). The use, distribution or reproduction in other forums is permitted, provided the original author(s) and the copyright owner(s) are credited and that the original publication in this journal is cited, in accordance with accepted academic practice. No use, distribution or reproduction is permitted which does not comply with these terms. 